\title{
Power Constrained High-Level Synthesis of Battery Powered Digital Systems
}

\author{
Nielsen, Sune Fallgaard; Madsen, Jan
}

Published in:

DATE 2003

Publication date:

2003

Document Version

Publisher's PDF, also known as Version of record

Link back to DTU Orbit

Citation (APA):

Nielsen, S. F., \& Madsen, J. (2003). Power Constrained High-Level Synthesis of Battery Powered Digital Systems. In DATE 2003 (pp. 1136-1137). IEEE.

\section{General rights}

Copyright and moral rights for the publications made accessible in the public portal are retained by the authors and/or other copyright owners and it is a condition of accessing publications that users recognise and abide by the legal requirements associated with these rights.

- Users may download and print one copy of any publication from the public portal for the purpose of private study or research.

- You may not further distribute the material or use it for any profit-making activity or commercial gain

- You may freely distribute the URL identifying the publication in the public portal

If you believe that this document breaches copyright please contact us providing details, and we will remove access to the work immediately and investigate your claim. 


\title{
Power Constrained High-Level Synthesis of Battery Powered Digital Systems
}

\author{
S.F. Nielsen and J. Madsen \\ Informatics and Mathematical Modelling \\ Technical University of Denmark \\ $\{$ sfn,jan\}@imm.dtu.dk
}

\begin{abstract}
We present a high-level synthesis algorithm solving the combined scheduling, allocation and binding problem minimizing area under both latency and maximum power per clock-cycle constraints. Our approach eliminates the large power spikes, resulting in an increased battery lifetime, a property of outmost importance for battery powered embedded systems. Our approach extends the partial-clique partitioning algorithm of [3] by introducing power awareness through a heuristic algorithm which bounds the design space to those of power feasible schedules. We have applied our algorithm on a set of dataflow graphs and investigated the impact on circuit area when applying different power constraints.
\end{abstract}

\section{Introduction}

Our target applications are low-cost portable embedded systems. Today, consumers demand portable applications so tiny that they go virtually undetected when not in use. An interesting aspect of this application area is the lowcost issue which puts focus on reducing the overall system cost, eg. a requirement to select a low-priced (low-quality) battery over a high-priced (high-quality) battery. Now, the amount of total energy/charge available from a battery, and thus its life-time, depends strongly on the current profile of the application $[1,2]$. In particular if the peak-current exceeds a maximum-threshold the life-time starts dropping dramatically, this effect is more dominant on batteries of low quality, where up to a 20-30 percent extension of lifetime has been reported when designing for battery powered systems [1]. In figure 1 is shown both an undesirable and a more desirable schedule.

So far the main efforts in low-power synthesis can be divided into two groups: (a) Low level allocation and assignment $[4,5,6,7]$ : Here the goal is to combine functional units and operations in such a way that the internal

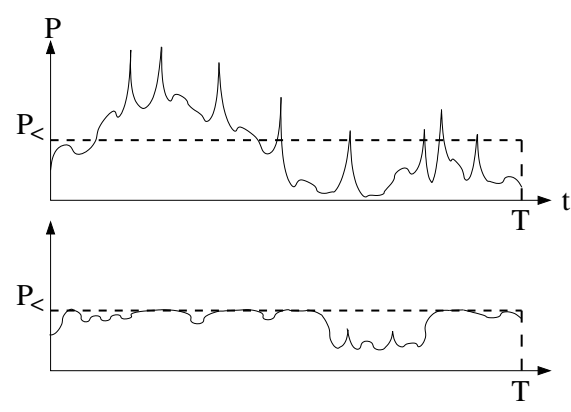

Figure 1. Undesired power schedule (top). Desired power schedule (bottom).

switching activity of the functional units is minimized. (b) Task level scheduling [1, 2, 8]: Here the goal is to schedule tasks in such an order the peak system power is minimized. The majority of these algorithms are either based on meta-heuristic algorithms, or two-step algorithms $[1,2]$ where in step one a traditional time constrained schedule is constructed and in step two the schedule is reordered to meet the power constraint.

In this paper we present a heuristic synthesis algorithm which solves: (i) scheduling, (ii) allocation and (iii) assignment simultaneously under both a time and power constraint. This enables us to expand the exploration of the design space to include different types of functional units eg. the speed and energy usage of an operator can be traded versus the area of the operator.

\section{Power Heuristic Scheduling}

The main idea of our algorithm is to heuristically "stretch" the classical asap schedule to fit the power constraint, ie. to schedule the operators as fast as possible, but only if there is power available meaning some operators will be delayed additional cycles. The power constrained asap 


\begin{tabular}{|l|l|r|r|r|}
\hline Module & Oprs & Area & Clk-cyc. & P \\
\hline \hline add & $\{+\}$ & 87 & 1 & 2.5 \\
\hline sub & $\{-\}$ & 87 & 1 & 2.5 \\
\hline comp & $\{>\}$ & 8 & 1 & 2.5 \\
\hline ALU & $\{+,-,>\}$ & 97 & 1 & 2.5 \\
\hline Mult (ser.) & $\{*\}$ & 103 & 4 & 2.7 \\
\hline Mult (par.) & $\{*\}$ & 339 & 2 & 8.1 \\
\hline input & imp & 16 & 1 & 0.2 \\
\hline output & xpt & 16 & 1 & 1.7 \\
\hline
\end{tabular}

Table 1. Functional unit library.

scheduling algorithm, pasap $\left(P_{<}\right)$, is as follows:

Initialize: Schedule source start-time to zero and initialize the execution offset $o_{i}$ (cycles) to zero for all operators.

step 1: Pick an unscheduled operator $v_{i}$

step 2: If $v_{i}$ has unscheduled predecessors, goto 4 .

step 3: if there is power available in the execution time interval $\left[\left(t_{i}+o_{i}\right) . .\left(t_{i}+o_{i}+d_{i}\right)\right]$, where $d_{i}$ is the execution delay of $v_{i}$ and $t_{i}=\max \left\{t_{j}+d_{j}\right\} \forall v_{j} \rightarrow v_{i}$, schedule operation $i$ at time $t_{i}$, otherwise increase $o_{i}$ by one.

step 4: If unscheduled operators, goto step 1.

We have enhanced the formulation of a valid "timeextendend compatibility graph" $(V 1)$ in [3] to include power constraints using the pasap and its time-revesed palap algorithms. Then the solution to the synthesis problem with the minimum area and using least interconnect is the problem of finding the Partial minimal cost clique partitioning of $V 1$ which does not violate the power constraint. As in [3] we will heuristically solve the clique partitioning problem, through a greedy approach ie. evaluate the $V 1$ graph and pick a "best" decision, which is then scheduled, allocated and assigned and then repeat the process until no operators are left. During this we need to ensure feasibility, as pasap and palap are heuristic algorithms they depend on what operators have been scheduled, therefore a sequence of assignments might cause the deletion of unscheduled operators, causing an invalid schedule. The solution is to backtrack one step and lock the start time of all unscheduled operators to the pasap schedule (which was valid) and then continue.

We have benchmarked the algorithm on some CDFGs, where we have investigated the area of the resulting circuits as a function of time and power constraints. The results are shown in figure 2. The FU library used in the tests is shown in table 1 .

\section{Conclusion}

In this paper we have presented an algorithm for time and power constrained synthesis of digital circuits. We have applied the algorithm on the traditional synthesis benchmark

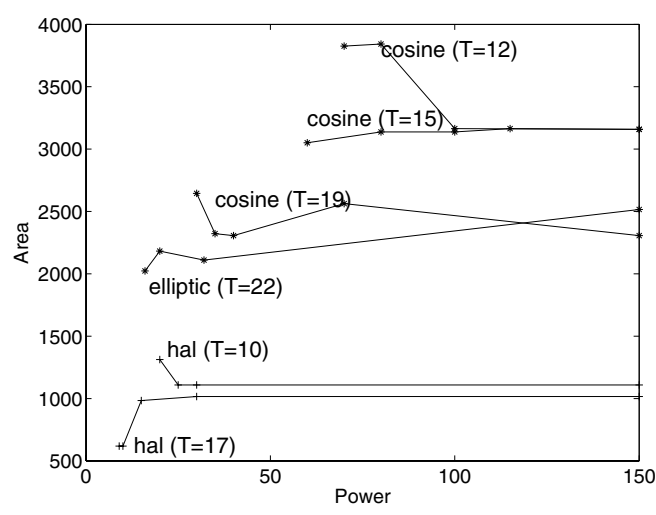

Figure 2. Power vs. area under different time constraints for our CDFGs.

sets and investigated different regions in the time-powerconstraint space. For our benchmarks we have found that we are able to trade in a small amount of area to obtain a solution which fits our power requirements.

\section{References}

[1] J. Luo and N. K. Jha. Battery-Aware Static Scheduling for Distributed Real-time Embedded Systems. In proceedings of 38th Design Automation Conference, 2001. Page(s): 444 -449 .

[2] K.Lahiri, A. Raghunathan, S. Dey. Communication Architecture Based Power Management for Battery Efficient System Design. In proceedings of Design, Automation and Test in Europe. 2002. Page(s): 691 -696

[3] J. Jou; S. Kuang; R. Chen. Clique Partitioning Based Integrated Architecture Synthesis for VLSI Chips. In proceedings of International Symposium on VLSI Technology, Systems, and Applications, 1993, Page(s): 58 -62.

[4] Y. Choi and T. Kim. An Efficient Low-Power Binding Algorithm in High-Level Synthesis. Circuits and Systems, 2002. ISCAS 2002. IEEE International Symposium on , Volume: 4, 2002 Page(s): $321-324$.

[5] M. S. Bright and T. Arslan. Synthesis of Low-Power DSP Systems Using a Genetic Algorithm. In IEEE Transactions on Evolutionary Computation, vol. 5, no. 1, Feb 2001.Page(s): $27-40$.

[6] F. Gruian and K. Kuchcinski. Operator Binding and Scheduling for Low Power Using Constraint Logic Programming. Euromicro Conference, 1998. Proceedings. 24th , Volume: 1, 1998 Page(s): 83 -90 vol.1.

[7] M. Bhardwaj, R. Min, A. Chandrakasan. Power-Aware Systems. Very Large Scale Integration (VLSI) Systems, IEEE Transactions on , Volume: 9 Issue: 6, Dec. 2001 Page(s): $757-772$.

[8] J. Liu, P. H. Chou, N. Bagherzadeh, F. Kurdahi. PowerAware Scheduling under Timing Constraint for MissionCritical Embedded Systems. In proceedings of the 38th Design Automation Conference, 2001. Page(s): 840 -845. 